\title{
The pursuit of normal reference values of pancreas stiffness by using Acoustic Radiation Force Impulse (ARFI) elastography
}

\author{
Razvan Zaro ${ }^{1}, 2$, Monica Lupsor-Platon ${ }^{2}$, Alexandre Cheviet ${ }^{2}$, Radu Badea ${ }^{2}$
}

${ }^{1}$ Gastroenterology Department, ${ }^{2}$ Department of Imaging and Radiology, $3^{\text {rd }}$ Medical Clinic, “Octavian Fodor” Regional Institute of Gastroenterology and Hepatology, "Iuliu Hatieganu" University of Medicine and Pharmacy, ClujNapoca, Romania

\begin{abstract}
Aim: The purpose of this study is to evaluate pancreatic stiffness by ARFI abdominal elastography. In the current literature, there are relatively few studies that have assessed the clinical utility of this technique. Material and method: A number of 37 healthy subjects were included. The data were collected in a prospective manner and afterwards included in an observational, analytical and longitudinal study. Subsequently viewing the pancreatic parenchyma in bidimensional mode (2D-US) mode, 10 shear wave velocity (SWV) measurements for each segment: head, body and tail were performed. Statistical analysis by regression models targeted also the possible influence of other factors in assessing SWV. A comparative analysis was performed regarding the statistical significance of 5 versus 10 SWV measurements for each segment. Results: The pancreas was entirely evaluated in all subjects. The mean SWV from the entire parenchyma was $1.216 \mathrm{~m} / \mathrm{s} \pm 0.36$ and between the three segments SWV were similar (head: $1.224 \mathrm{~m} / \mathrm{s}$, body: $1.227 \mathrm{~m} / \mathrm{s}$ and tail: $1.191 \mathrm{~m} / \mathrm{s}$ ). A ratio of the IQR/Median $>0.4$ was interpreted as statistically invalid, relevant data being highlighted in the percentage of $83.78 \%$ for the head of the pancreas, $78.37 \%$ for the body, and $67.56 \%$ at the caudal level. Significant correlations were observed between the data (mean and median SWV) provided by the group with 5 measurements of the SWV versus the standard group: $93.9 \%$ for the head, $96.6 \%$ for the body, and $98.7 \%$ accordingly to the tail. Conclusions: SWV determination by percutaneous approach represents a useful imaging method for evaluating pancreatic stiffness, of course within these limitations. Because we did not observe statistically significant differences between the results obtained by 5 or 10 measurements, we suggest that it would be sufficient to perform only five measurements of the SWV per pancreatic segment. The data obtained in the normal pancreas could be used in future comparative assessments regarding the inflammatory or tumoral pathology of the pancreas.
\end{abstract}

Keywords: pancreas, percutaneous elastography, ROI, VTQ, SWV.

\section{Introduction}

Abdominal ultrasonography (US) is the imaging method used per primam in pancreatic evaluation and related pathology (inflammatory and tumoral), being considered as a non-invasive, reproducible and accuracy technique [1]. Elastography is an imaging technique for assessing tissue stiffness with extensive applications in the evaluation of the breast, thyroid, lymph nodes, and

Received 18.06.2016 Accepted 01.08.2016

Med Ultrason

2016, Vol. 18, No 4, 425-430

Corresponding author: Răzvan Zaro

$3^{\text {rd }}$ Medical Clinic,

19-21 Croitorilor Street,

400162, Cluj Napoca, România

E-mail: Razvan.Zaro@umfcluj.ro skin [2]. There are various elastography techniques, one of them being elastography by a pulse of acoustic radiation (ARFI) method [3].

Using ARFI, qualitative and quantitative determination of the tissue stiffness can be obtained. This is technically feasible with acoustic emission pulses within a region of interest (ROI) that produce a tissue displacement that will generate acoustic shear waves (SW) [4]. Both local impact of the acoustic pulses and also the data regarding the SW that are generated, propagated, and received are framed into a function of the elastic modulus (Young's modulus) $[5,6]$. The values of this function will represent SW velocity (SWV) at the ROI level, a velocity that is directly proportional with expressing the tissue stiffness. SW may come from a variety of sources including an acoustic pulse generated by a radiation and can be generated in the tis- 
sue by applying a directional force [3]. A high SWV is found in the stiffer tissue $[7,8]$. There are several methods for performing SW elastography (SWE): 2D-SWE, point SWE, and transient elastography (TE) [9-12].

ARFI imaging technique through Virtual Touch Tissue Quantification (VTQ) software (Virtual Touch ${ }^{\mathrm{TM}}$ - Tissue - Quantification, Siemens ACUSON - S2000, Mountain View, CA, USA) produces SW secondary to mechanical excitations within the ROI. Subsequently, $\mathrm{SW}$ is propagated in the ROI and detected and analyzed according to correlation algorithms [4]. Implementation of the ARFI technique in evaluating liver stiffness has been evidenced in numerous studies [13-17]. The fact that ARFI can be coordinated under the 2D-US guidance is a real advantage.

Point SWE has proven diagnostic assessment in liver fibrosis in patients with chronic viral hepatitis B and C, alcoholic and non-alcoholic steatohepatitis [18-21]. Also, the ARFI technique is used in the assessment of liver fibrosis, in the pathology of the thyroid, breast, prostate, and lymph nodes [9, 22-26].

At this moment only a few studies that have assessed the normal range value for pancreatic stiffness have been published [27-30]. This study will add a contribution to the already published data, by virtue of the necessity for standard values when discussing pathology. This prospective study aims to assess stiffness of the healthy pancreas by the transabdominal ARFI elastography technique.

\section{Material and method}

The study was conducted prospectively in a longitudinal manner. All investigations were performed by a single operator after the informed consent of patients was obtained. Local Ethics Committee approval was obtained.

\section{Patients}

The rated group comprised 37 healthy subjects, all of them volunteers. Inclusion criteria for the study were: subjects with no history of pancreatic pathology (chronic pancreatitis, diabetes, pancreatic tumours), normal weight (which is corresponding to a body mass index [BMI] range of $18.5-24.9 \mathrm{~kg} / \mathrm{m}^{2}$ ), and without chronic consumption of toxic substances (alcohol and/or tobacco). Subjects were excluded from the study with one of the following: non-visualisation of the pancreas in 2DUS examination (obesity, pancreatectomy, or increased bloating), medial-abdominal scars, history of tumor or radiotherapy in the area of the pancreas, gastro-duodenal prostheses, or gastro-jejunostomy.

\section{Ultrasound and elastography evaluation}

US and elastography exploration were performed using a $6 \mathrm{C} 1$ convex transducer with a frequency between

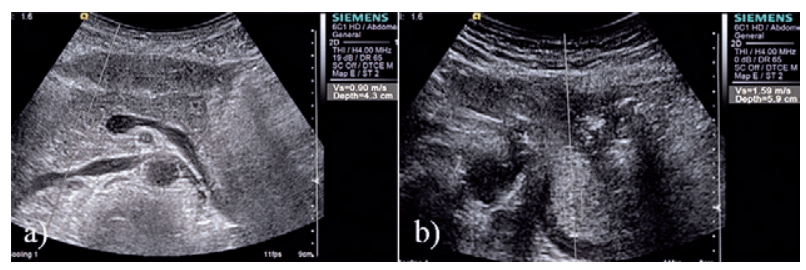

Fig 1. Transabdominal measurement of SWV by ARFI-SWV at the cephalic (a) and caudal (b) level. Enclosed in the SWV expressed in $\mathrm{m} / \mathrm{s}$ is displayed the depth of the ROI

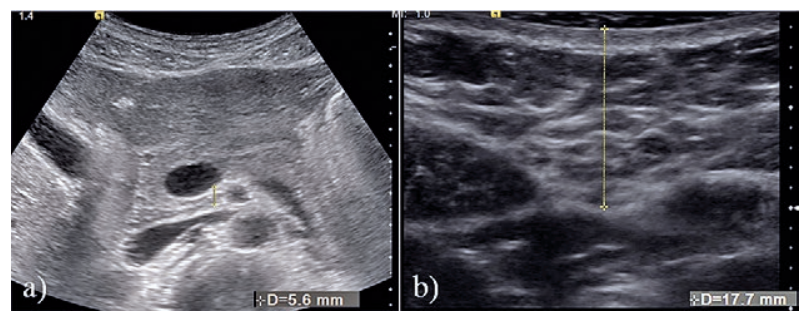

Fig 2. a) The size of adiposity adjoining the SMA is measured within the following anatomic relationships: right side to SMA (within $5-10 \mathrm{~mm}$ ), posterior to splenoportal confluence and anterior to the left renal vein; b) The parietal thickness was measured from linea alba to the external visible layer of the skin tissue in the area corresponding topographically to the pancreas.

1.5 to $6 \mathrm{MHz}$ (Siemens Acuson S2000. Siemens Medical Solutions, Mountain View, CA, USA), with the patient positioned supine, after a 12 hour fasting period. The transducer was applied with a minimum pressure, the subject being asked to avoid deep breathing during the examination.

The quantitative analysis was conducted through VTQ software that had allowed measurement of SWV in real time. At first, a 2D-US assessment of the pancreatic parenchyma in all three segments was achieved: head, body, and tail. Then the ROI window with dimensions of $5 / 10 \mathrm{~mm}$ was placed in the pancreatic tissue, without contact with the liver parenchyma, adjacent vessels, or structures of the digestive tract. After positioning the ROI window, an ARFI - acoustic impulse was triggered. In a very short time (about 1-2 seconds), SWV was displayed in $\mathrm{m} / \mathrm{s}$, along with the depth of the ROI placement (fig 1). For each segment of the pancreas 10 measurements were performed. We used transverse or slightly oblique transverse sections. For each subject we used a comparative model between the median (M) and average (m) of 5 versus 10 SWV measurements for each pancreatic segment. Data report regarding the interquartile range (IQR)/Median ratio $>0.4(40 \%)$ were excluded as statistically invalid [31-35].

The anterior-posterior diameter of each segment of pancreas was measured. Echogenicity of the pancreas was evaluated in comparison with the parenchyma of the 
left liver lobe, both overall and for each segment being classified as hypo- (1), iso- (2), or hyperechoic (3). Bloating was quantified as moderate/heavy (1) or minimum (0), after gradual transducer compression of air at this level. The thicknesses of adiposity adjoining the superior mesenteric artery (SMA) (fig 2a) and of the subcutaneous tissue at the epigastric level (fig 2b) were measured in order to evaluate their potential effect on pancreatic tissue stiffness. For some subjects, a progressive pressure with the transducer was needed to favor the displacement of intestinal air, which sometimes obstructed a proper caudal view. For the measurement of the subcutaneous tissue thickness we used a linear 9L4 transducer with a nominal frequency between 4 to $9 \mathrm{MHz}$.

\section{Statistical analysis}

The data were processed using Excel 2016 for the Windows 10 and IBM Statistical Program for Social Sciences (SPSS) Version 20 software. Elements of descriptive statistics such as mean, median and standard deviation (SD) were used. Simple and multiple linear regression among bivariate correlation (Pearson's correlation coefficient) were applied for the relation between SWV values and the rest of the independent variables (abdominal parietal thickness, perivisceral adiposity, BMI, abominable bloating, parenchymal echogenicity, size of the pancreatic segments, depth of the ROI, and age). The $t-$ Test was applied among average and median values between the two groups of SWV measurements: 5 versus 10 measurements. A p value less than 0.05 was interpreted as statistically valid. For every participant, the mean, median, SD and IQR from the corresponding ROI in each of the pancreatic segment were calculated.

\section{Results}

In the study group ( 37 healthy subjects), 15 were men $(40.5 \%)$, aged between 21 and 33 years, with BMI between 16.08 and $25.20 \mathrm{~kg} / \mathrm{m}^{2}\left(20.54 \pm 2.03 \mathrm{~kg} / \mathrm{m}^{2}\right)$. Dimensions of the pancreatic segments are detailed in Table I.
The ratio IQR/Median was less than 0.4 in $83.78 \%$ of the measurements performed at the cephalic level, in $78.37 \%$ for the corporeal segment and in $67.56 \%$ respectively at the tail level. In fig 3 the outliers from the mean depth of the ROI measurements regarding each segment can be seen.

The stiffness analysis corresponding to the cephalic, body and tail level is expressed in Table II; the average SWV and measurements success rate are highlighted, with the mention that for the cephalic segment 31 subjects were taken into account; for the corporeal 29 and the tail of the pancreas valid measurements in 24 subjects were evidenced. In terms of cumulative average SWV for the entire pancreas a value of $1.216 \pm 0.36 \mathrm{~m} / \mathrm{s}(\mathrm{M}=1.10)$ was depicted.

In fig 4 the outliers from the mean SWV values regarding each segment can be seen. In $19(51.4 \%)$ subjects

Table I. Dimensions of the pancreatic segments ( $\mathrm{mm})$.

\begin{tabular}{lll}
\hline & Mean \pm SD & Median (25th-75th percentile) \\
\hline Head & $21.26 \pm 4.4$ & $21.3(11.5-30.7)$ \\
Body & $16.48 \pm 4.25$ & $16(8.1-28.4)$ \\
Tail & $20.14 \pm 3.1$ & $20.2(13.7-28.3)$ \\
\hline
\end{tabular}

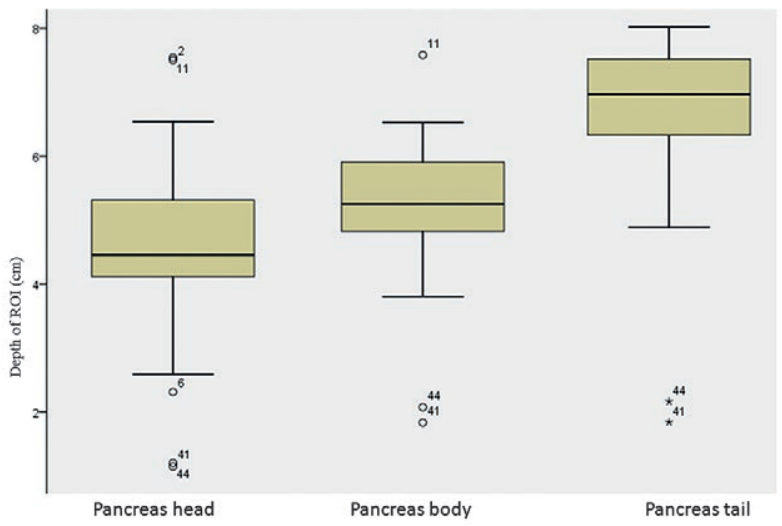

Fig 3. The mean depth $(\mathrm{cm})$ of the ROI
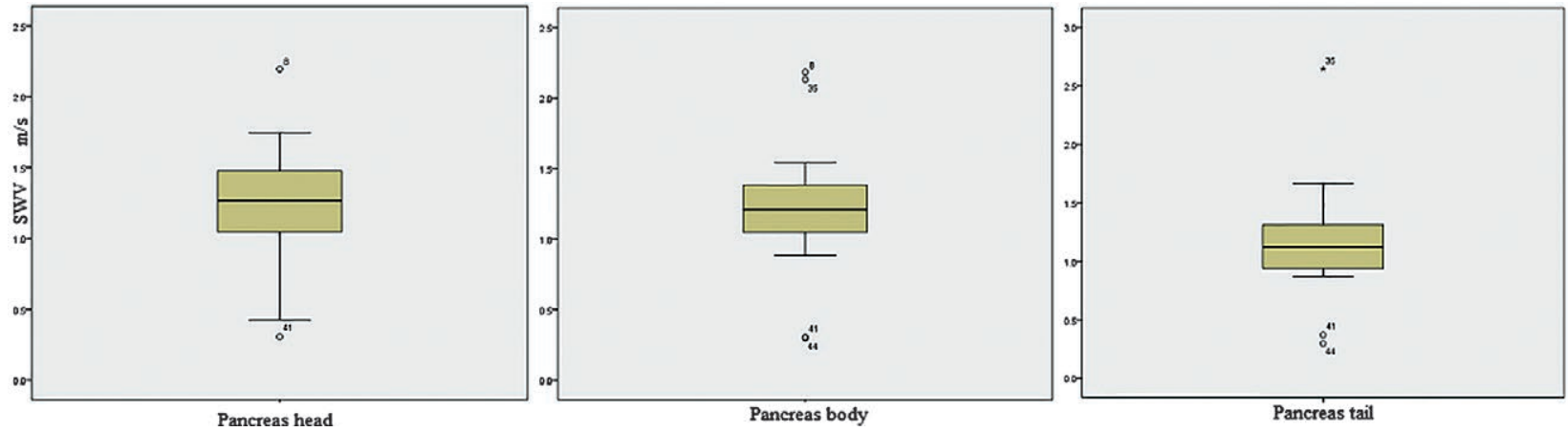

Fig 4. The mean SWV $-\mathrm{m} / \mathrm{s}$, regarding each pancreatic segment 
Table II. The SWV values for every segment of the pancreas and success rate on available SWV measurements

\begin{tabular}{|c|c|c|c|c|c|c|c|c|c|c|c|c|}
\hline \multicolumn{7}{|c|}{ SWV - 10 measurements } & \multicolumn{6}{|c|}{ SWV - 5 measurements } \\
\hline & $\begin{array}{l}\text { Number } \\
\text { of subjects }\end{array}$ & $\begin{array}{l}\text { Interval } \\
\mathrm{m} / \mathrm{s}\end{array}$ & $\begin{array}{l}\text { Mean } \pm \text { SD } \\
\mathrm{m} / \mathrm{s}\end{array}$ & Median & $\begin{array}{l}\text { IQR/ } \\
\text { Median }\end{array}$ & $\begin{array}{l}\text { Succes } \\
\text { Rate }\end{array}$ & $\begin{array}{l}\text { Number } \\
\text { of subjects }\end{array}$ & $\begin{array}{l}\text { Interval } \\
\mathrm{m} / \mathrm{s}\end{array}$ & $\begin{array}{l}\text { Mean } \pm \text { SD } \\
\mathrm{m} / \mathrm{s}\end{array}$ & Median & $\begin{array}{l}\text { IQR/ } \\
\text { Median }\end{array}$ & $\begin{array}{l}\text { Succes } \\
\text { Rate }\end{array}$ \\
\hline Head & 31 & $0.84-2.3$ & $1.224 \pm 0.33$ & 1.14 & 0.39 & $93 \%$ & 32 & $0.76-2.12$ & $1.234 \pm 0.32$ & 1.15 & $0.46^{*}$ & $92.2 \%$ \\
\hline Body & 29 & $0.87-2.29$ & $1.227 \pm 0.34$ & 1.11 & 0.22 & $97.2 \%$ & 34 & $0.77-2.29$ & $1.211 \pm 0.30$ & 1.15 & 0.31 & $97 \%$ \\
\hline Tail & 24 & $0.84-2.97$ & $1.191 \pm 0.43$ & 1.05 & 0.24 & $91.3 \%$ & 25 & $0.84-3.08$ & $1.250 \pm 0.47$ & 1.13 & 0.32 & $94.4 \%$ \\
\hline
\end{tabular}

* data were outside of the statistically valid echelon, SD - standard deviation, IQR - interquartile range

Table III. Influence of independent variables on SWV

\begin{tabular}{llll}
\hline Independent variable & Head & Body & Tail \\
\hline Adiposity near the SMA & 0.559 & 0.992 & 0.234 \\
BMI & 0.640 & 0.646 & 0.599 \\
Abdominal bloating & 0.939 & 0.977 & 0.703 \\
Echogenicity of the pancreas & 0.538 & 0.456 & 0.303 \\
Anterior-posterior diameter at caudal level & 0.837 & 0.322 & 0.803 \\
Depth of the ROI & $\mathbf{0 . 2 2 6}\left(\mathbf{r}^{2}=. \mathbf{2 0 6}\right)$ & $\mathbf{0 . 1 1 9}\left(\mathbf{r}^{\mathbf{2}=. \mathbf{0 0 0})}\right.$ & 0.807 \\
Age & 0.660 & 0.941 & 0.764 \\
Abdominal wall thickness & 0.538 & 0.104 & $\mathbf{0 . 1 8 2}\left(\mathbf{r}^{2}=\mathbf{. 0 0 0}\right)$ \\
\hline
\end{tabular}

BMI - body mass index, SMA - superior mesenteric artery

Table IV. SWV comparative values in the two groups: 5 and 10 measurements and correlations between the data provided by them.

\begin{tabular}{|c|c|c|c|c|c|c|}
\hline \multicolumn{3}{|c|}{ SWV - 10 measurements } & \multicolumn{4}{|c|}{ SWV - 5 measurements } \\
\hline & Mean $\mathrm{m} / \mathrm{s}$ & Median & Mean $\mathrm{m} / \mathrm{s}$ & Median & Corelation & Interval, 95\% CI \\
\hline Head & 1.224 & 1.14 & 1.234 & 1.15 & $93.9 \%$ & [71.6-99.6] \\
\hline Body & 1.227 & 1.11 & 1.211 & 1.15 & $96.6 \%$ & [93.1-99.6] \\
\hline Tail & 1.191 & 1.05 & 1.250 & 1.13 & $98.7 \%$ & {$[95.8-99.7]$} \\
\hline
\end{tabular}

the abdominal bloating was interpreted as minimum. Average adiposity layer adjacent to the SMA was $6.36 \pm 1.52$ $\mathrm{mm}(\mathrm{M}=6)$ and the subcutaneous tissue $14.03 \pm 4.31 \mathrm{~mm}$ $(\mathrm{M}=14.5)$.

In multiple linear regression analysis, the ROI depth was the only independent variable that influenced SWV in the cephalic segment, but without a statistical significance. At the body of the pancreas, SWV values are influenced in a percentage of $11.7 \%$ by the depth of the ROI. Regarding data for the tail of the pancreas, the only external variable that could influence the values of the SWV turned out to be the abdominal wall thickness (Table III).

\section{Data regarding 5 measurements per segment}

We performed an analysis regarding the accuracy of the data provided by a model with only 5 SWV valid measurements. In this model the average successful measurements were $4.61 \pm 0.65$ for the cephalic segment $(\mathrm{M}=5), 4.85 \pm 0.035$ in the corporeal level $(\mathrm{M}=5)$ and $4.72 \pm 0.6$ for the pancreatic tail $(M=5)$.

The number of evaluable subjects in the group with 5 SWV measurements per segment was similar to those included in the group of 10 measurements. Using the T-test and the same IQR/Median ratio we noticed that descrip- tive statistical data between the groups with 5 versus 10 measurements per segment were overlapping (Table IV).

\section{Discussions}

The similarity of the mean and median values regarding SWV confirms the uniformity of distributed data. A previous study indicates a directly proportional relationship between the stiffness of the tissue and SWV [36]. SWV values for the healthy pancreas $(1.216 \mathrm{~m} / \mathrm{s} \pm 0.36)$ are similar to those obtained by other authors. Kawada et al achieved a slightly higher average of the SWV of $1.43 \pm 0.28 \mathrm{~m} / \mathrm{s}$ [33]. Mateen et al detected a mean SWVVTQ value of $1.28 \mathrm{~m} / \mathrm{s}$ [34].

It is worth mentioning that Kawada et al used in their study also a 10 measurements/segment, but the number of healthy volunteers was less. The slightly higher mean SWV could be explained by age: $65 \pm 14$ years, non-pancreatic diseases (chronic hepatitis and hepatocellular carcinoma) and pancreatic diseases (pancreatic cysts) [33]. Our study included 37 apparently healthy volunteers with an age range between 21 and 33 years, normal BMI, and without any subsequent pancreatic disease. 
Mateen et al included in their study a larger healthy control group (52 subjects) with a similar age range (39.4 \pm 19.7 years), but a total number of 10 measurements were included, divided almost equally between pancreatic segments ( 3 measurements in the head and tail, and 4 for the body) [34].

All of the three studies $[33,34]$ including ours, used the same ultrasound gear (Siemens ACUSON - S2000 with VTQ software).

We used multiple linear regression in the analysis of the factors that could influence independently the SWV at each pancreatic segment: adiposity near the SMA, the thickness of the abdominal wall at the epigastric level, BMI, abdominal bloating, echogenicity of the pancreas, the anterior-posterior diameter of each segment and the depth where ROI was positioned.

From these factors, we surmised that only the depth of the ROI could influence pancreatic SWV values, and this is statistically significant only at the head of the pancreas.

Average and median SWV between the three pancreatic segments are similar in value. We also did not reveal statistically significant differences in the case of comparative analysis between the data provided by 5 versus 10 measurements per segment. Thus, we can assume that 5 measurements would be sufficient to determine accurately the pancreatic stiffness by percutaneous ARFI. In the current literature, data regarding percutaneous ARFI evaluation of the pancreas are related to 10 measurements/segment. In one study [34] minimum number of 3 measurements per segment were used, with similar results regarding the SWV for the normal pancreas.

Relevance of normal reference values lies in the necessity to proper interpret stiffness variations identified by the ARFI technique: a) as a complementary method of diagnosis of acute pancreatitis with a sensitivity (Se) and specificity $(\mathrm{Sp})$ of $100 \%$ and $98 \%$ (cutoff value of $\mathrm{SWV}$ was $1.63 \mathrm{~m} / \mathrm{s}$ ) [31]; b) regarding chronic pancreatitis, when a cutoff value of SWV of $1.40 \mathrm{~m} / \mathrm{s}$ for parenchymal stiffness was significantly increased compared to the healthy pancreas (AUROC $=0.78$ ) [30]; and c) cystic pancreatic pathology was also evaluated in a study aimed at differentiating between mucinous and serous cysts $(\mathrm{Se}=37.5 \%-68.8 \%, \mathrm{Sp}=77.3 \%-100 \%, \mathrm{PPV}=68.8 \%-100 \%$, and NPV $=68.8 \%-77.3 \%$ ) $[27,29]$.

Findings of pancreatic tissue stiffness by addressing SWE/ARFI percutaneous is limited at this time, as the VTQ software allows a maximum ROI depth up to $8 \mathrm{~cm}$. This, as has been shown, is not an impediment in subjects with normal BMI, but in obese patients could hinder the SWV measuring performance. Abdominal bloating can obstruct in 2D-US mode pancreatic viewing.
This study has some limitations. First, the group was formed only from younger subjects, the age range was quite narrow, and therefore extensive assessments regarding the relationship of age and pancreatic rigidity could not be done. A number of subjects proved to be enough to empower the study, but a major limit of the study was represented by the absence of biochemical confirmation that the group was composed of healthy subjects. The data provided by subjects relating to alcohol consumption and cigarette smoking were not sufficient to permit a statistical significant appreciation.

\section{Conclusions}

SWV measurement by percutaneous ARFI technique represents a useful imaging method in evaluating the rigidity of the pancreatic parenchyma entirely. The average value of shear wave velocity in the pancreas does not differ significantly between pancreatic segments. The data obtained in the normal pancreas can be used in the future in a comparative assessment of pancreatic inflammatory or tumor pathology. Implications regarding the diagnostic value represents the topic of subsequent studies.

\section{Conflict of interest: none}

\section{References}

1. Dimcevski G, Erchinger FG, Havre R, Gilja OH. Ultrasonography in diagnosing chronic pancreatitis: new aspects. World J Gastroenterol 2013;19:7247-7257.

2. Uomo G. Ultrasound elastography. A possible improvement into the paraphernalia of pancreatic imaging. JOP 2008;9:666-667.

3. Nightingale K, Soo MS, Nightingale R, Trahey G. Acoustic radiation force impulse imaging: in vivo demonstration of clinical feasibility. Ultrasound Med Biol 2002;28:227-235.

4. Bamber J, Cosgrove D, Dietrich CF, et al. EFSUMB guidelines and recommendations on the clinical use of ultrasound elastography. Part 1: Basic principles and technology. Ultraschall Med 2013;34:169-184.

5. McAleavey SA, Menon M, Orszulak J. Shear-modulus estimation by application of spatially-modulated impulsive acoustic radiation force. Ultrason Imaging 2007;29:87104.

6. Nightingale KR, Bentley R, Trahey G. Observations of tissue response to acoustic radiation force: opportunities for imaging. Ultrason Imaging 2002;24:129-138.

7. Castéra L, Vergniol J, Foucher J, et al. Prospective comparison of transient elastography, Fibrotest, APRI, and liver biopsy for the assessment of fibrosis in chronic hepatitis $\mathrm{C}$. Gastroenterology 2005;128:343.

8. Shiina T, Nightingale KR, Palmeri ML, et al. WFUMB guidelines and recommendations for clinical use of ultra- 
sound elastography: Part 1: basic principles and terminology. Ultrasound Med Biol 2015;41:1126-1147.

9. Talwalkar JA, Kurtz DM, Schoenleber SJ, West CP, Montori VM. Ultrasound based transient elastography for the detection of hepatic fibrosis: systematic review and metaanalysis. Clin Gastroenterol Hepatol 2007;5:1214-1220.

10. Sporea I, Sirli R, Bota S, Popescu A, Sendroiu M, Jurchis A. Comparative study concerning the value of acoustic radiation force impulse elastography (ARFI) in comparison with transient elastography (TE) for the assessment of liver fibrosis in patients with chronic hepatitis B and C. Ultrasound Med Biol 2012;38:1310-1316.

11. Lupsor M, Badea R, Stefanescu H, et al. Performance of a new elastographic method (ARFI technology) compared to unidimensional transient elastography in the noninvasive assessment of chronic hepatitis C. Preliminary results. J Gastrointestin Liver Dis 2009;18:303-310.

12. Dietrich CF, Cantisani V. Current status and perspectives of elastography. Eur J Radiol 2014;83:403-404.

13. Friedrich-Rust M, Nierhoff J, Lupsor M, et al. Performance of acoustic radiation force impulse imaging for the staging of liver fibrosis: a pooled meta-analysis. J Viral Hepat 2012;19:e212-e219.

14. Goertz RS, Amann K, Heide R, et al. An abdominal and thyroid status with acoustic radiation force impulse elastometry — a feasibility study: acoustic radiation force impulse elastometry of human organs. Eur J Radiol 2011;80:e226-e230.

15. Piscaglia F, Salvatore V, Di Donato R, et al. Accuracy of Virtual Touch Acoustic Radiation Force Impulse (ARFI) imaging for the diagnosis of cirrhosis during liver ultrasonography. Ultraschall Med 2011;32:167-175.

16. Karlas T, Pfrepper C, Wiegand J, et al. Acoustic radiation force impulse imaging (ARFI) for noninvasive detection of liver fibrosis: examination standards and evaluation of interlobe differences in healthy subjects and chronic liver disease. Scand J Gastroenterol 2011;46:1458-1467.

17. Yoon KT, Lim SM, Park JY, et al. Liver stiffness measurement using acoustic radiation force impulse (ARFI) elastography and effect of necroinflammation. Dig Dis Sci 2012;57:1682-1691.

18. Friedrich-Rust M, Wunder K, Kriener S, et al. Liver fibrosis in viral hepatitis: noninvasive assessment with acoustic radiation force impulse imaging versus transient elastography. Radiology 2009;252:595-604.

19. Friedrich-Rust M, Buggisch P, de Knegt RJ, et al. Acoustic radiation force impulse imaging for non-invasive assessment of liver fibrosis in chronic hepatitis B. J Viral Hepat 2013;20:240-247.

20. Friedrich-Rust M, Romen D, Vermehren J, et al. Acoustic radiation force impulse imaging and transient elastography for non-invasive assessment of liver fibrosis and steatosis in NAFLD. Eur J Radiol 2012;81:e325-e331.

21. Zhang D, Li P, Chen M, et al. Non-invasive assessment of liver fibrosis in patients with alcoholic liver disease using acoustic radiation force impulse elastography. Abdom Imaging 2015;40:723-729.
22. Lyshchik A, Higashi T, Asato R, et al. Thyroid gland tumor diagnosis at US elastography. Radiology 2005;237:202211.

23. Pallwein L, Mitterberger M, Pinggera G, et al. Sonoelastography of the prostate: Comparison with systematic biopsy findings in 492 patients. Eur J Radiol 2008;65:304310.

24. Lyshchik A, Higashi T, Asato R, et al. Cervical lymph node metastases: diagnosis at sonoelastography--initial experience. Radiology 2007;243:258-267.

25. Rubin JM, Aglyamov SR, Wakefield TW, O'Donnell M, Emelianov SY. Clinical application of sonographic elasticity imaging for aging of deep venous thrombosis: preliminary findings. J Ultrasound Med 2003;22:443-448.

26. Hamidi C, Göya C, Hattapoğlu S, et al. Acoustic Radiation Force Impulse (ARFI) imaging for the distinction between benign and malignant thyroid nodules. Radiol Med 2015;120:579-583.

27. D’Onofrio M, Gallotti A, Martone E, Pozzi Mucelli R. Solid appearance of pancreatic serous cystadenoma diagnosed as cystic at ultrasound acoustic radiation force impulse imaging. JOP 2009; 10:543-546.

28. Garra BS. Imaging and estimation of tissue elasticity by ultrasound. Ultrasound Q 2007;23:255-268.

29. D'Onofrio M, Crosara S, Canestrini S, et al. Virtual analysis of pancreatic cystic lesion fluid content by ultrasound acoustic radiation force impulse quantification. J Ultrasound Med 2013;32:647-651.

30. Yashima Y, Sasahira N, Isayama $H$, et al. Acoustic radiation force impulse elastography for noninvasive assessment of chronic pancreatitis. J Gastroenterol 2012;47:427-432.

31. Goya C, Hamidi C, Hattapoglu S, et al. Use of acoustic radiation force impulse elastography to diagnose acute pancreatitis at hospital admission: comparison with sonography and computed tomography. J Ultrasound Med 2014;33:1453-1460.

32. Saftoiu A, Vilmann P, Gorunescu F, et al. Neural network analysis of dynamic sequences of EUS elastography used for the differential diagnosis of chronic pancreatitis and pancreatic cancer. Gastrointest Endosc 200;68:10861094.

33. Kawada N, Tanaka S, Uehara H, et al. Potential use of point shear wave elastography for the pancreas: A single-center prospective study. Eur J Radiol 2014;83:620-624.

34. Mateen MA, Muheet KA, Mohan RJ, et al. Evaluation of ultrasound based acoustic radiation force impulse (ARFI) and eSie touch sonoelastography for diagnosis of inflammatory pancreatic diseases. JOP 2012;13:36-44.

35. Cassinotto C, Lapuyade B, Ait Ali A, et al. Liver fibrosis: noninvasive assessment with acoustic radiation force impulse elastography - comparison with FibroScan M and XL probes and FibroTest in patients with chronic liver disease. Radiology 2013;269:283-292.

36. Nightingale K, McAleavey S, Trahey G. Shear-wave generation using acoustic radiation force: in vivo and ex vivo results. Ultrasound Med Biol 2003;29:1715-1723. 